Electronic Supplementary Information.

The 5-Dehydro-m-Xylylene Triradical and Its Nitrogen and Phosphorus Derivatives: Open-Shell Doublet versus Quartet

\title{
Ground State
}

Hue Minh Thi Nguyen, ${ }^{\text {a,b }}$ G. Gopakumar ${ }^{\mathrm{a}}$ and Minh Tho Nguyen ${ }^{\mathrm{a}, *}$ 
Figure 1S.

The active orbitals of $\mathrm{C}_{8} \mathrm{H}_{8}$ and its radical cation at CASSCF/ANO-L.
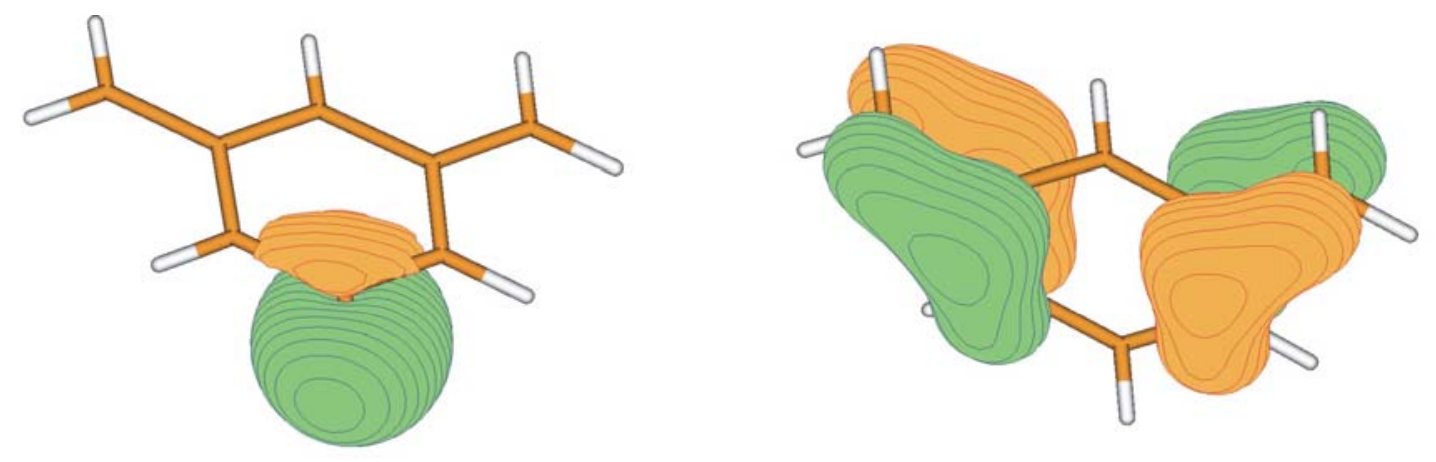

\section{$14 a_{1}$}
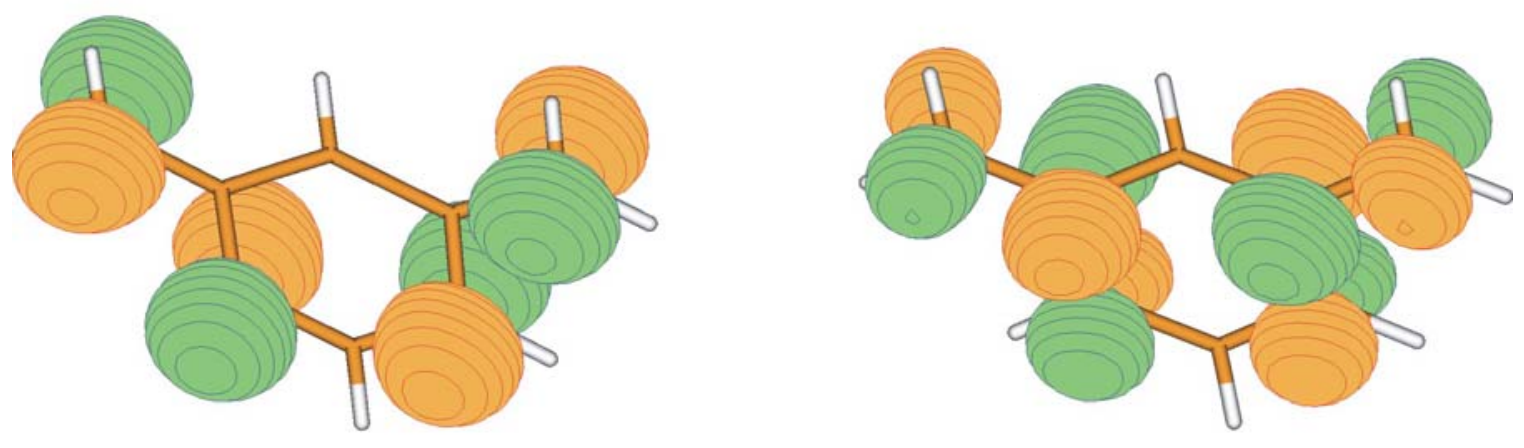

$2 a_{2}$

$3 a_{2}$ 
Figure 1S. (Continued)
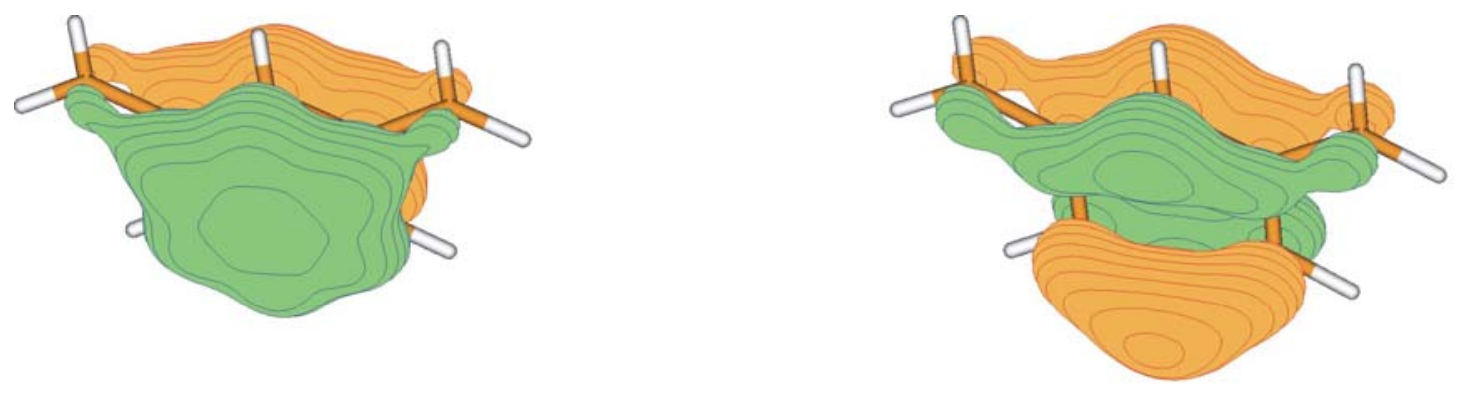

$1 b_{1}$

$2 b_{1}$
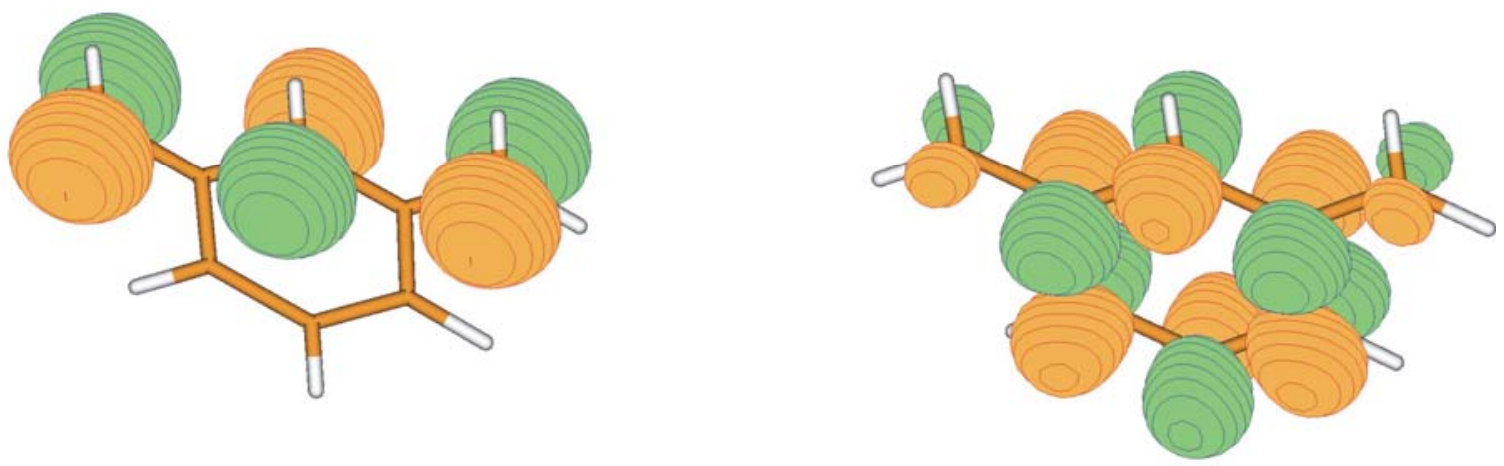

$3 b_{1}$

$4 b_{1}$

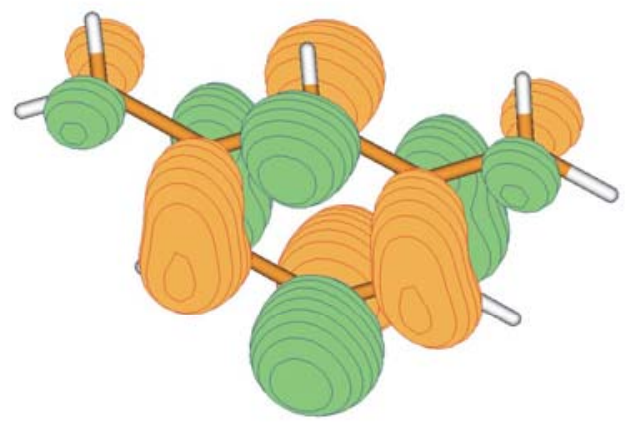

$5 b_{1}$ 
Figure 2S.

The active orbitals of $\mathrm{C}_{8} \mathrm{H}_{7}$ at CASSCF/ANO-L.

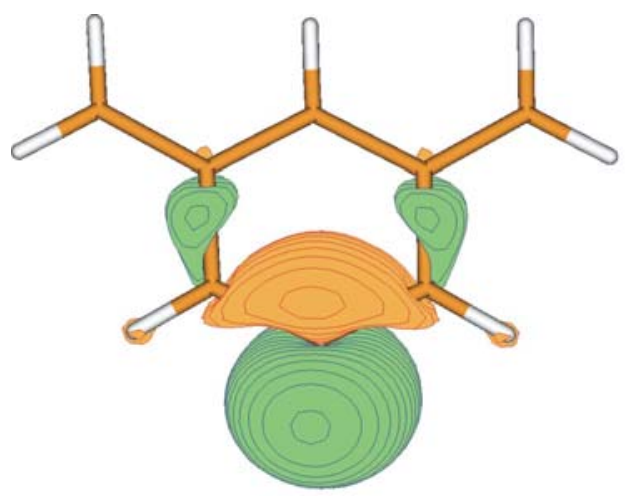

$14 \mathrm{a}_{1}$

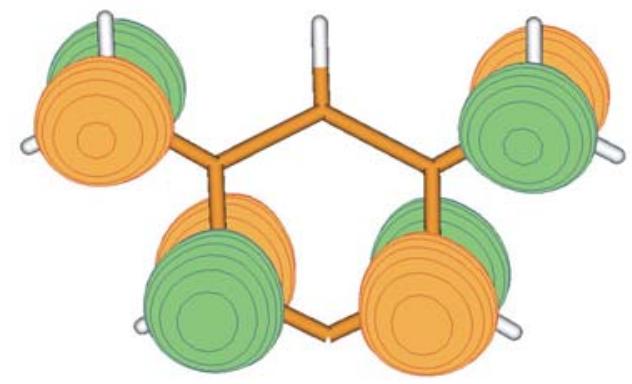

$2 \mathrm{a}_{2}$

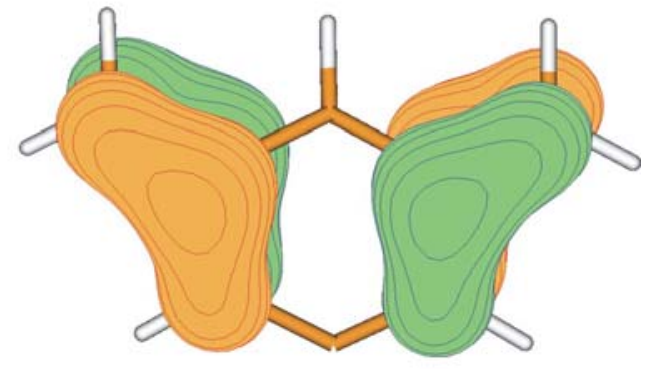

$1 \mathrm{a}_{2}$

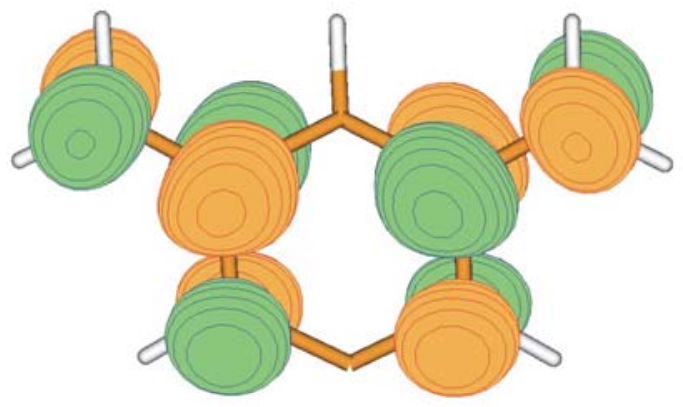

$3 \mathrm{a}_{2}$ 
Figure 2S. (Continued)
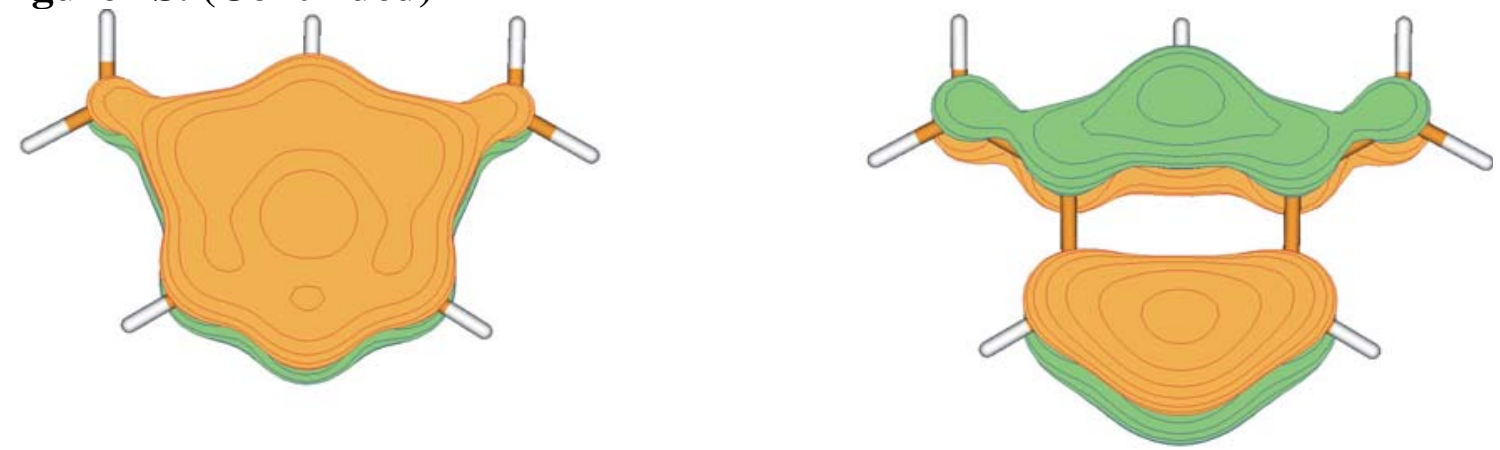

$1 b_{1}$
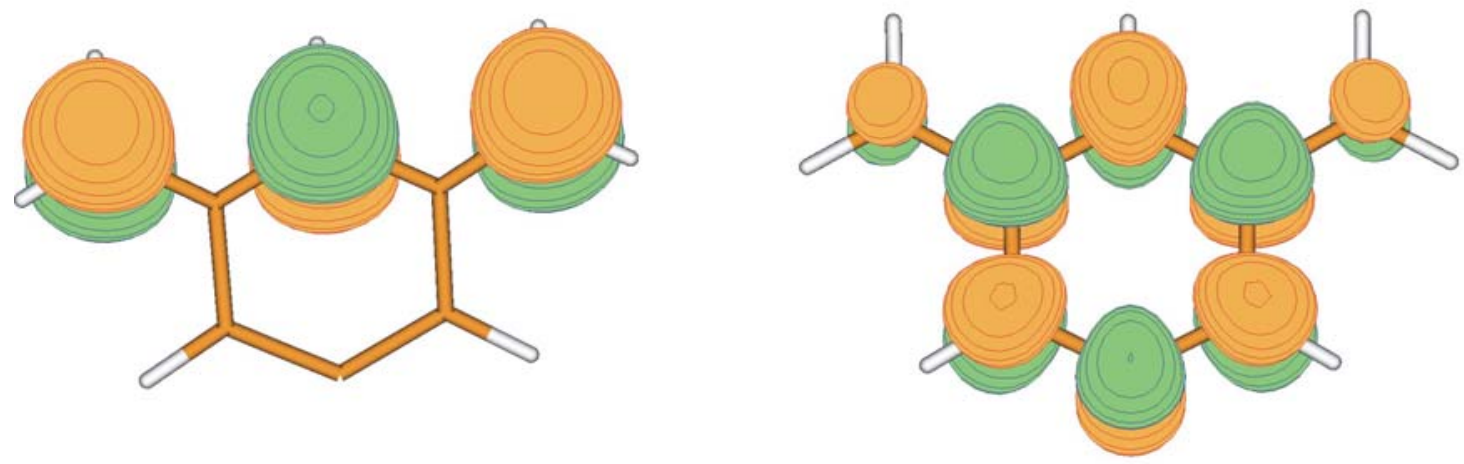

$3 b_{1}$

$4 b_{1}$

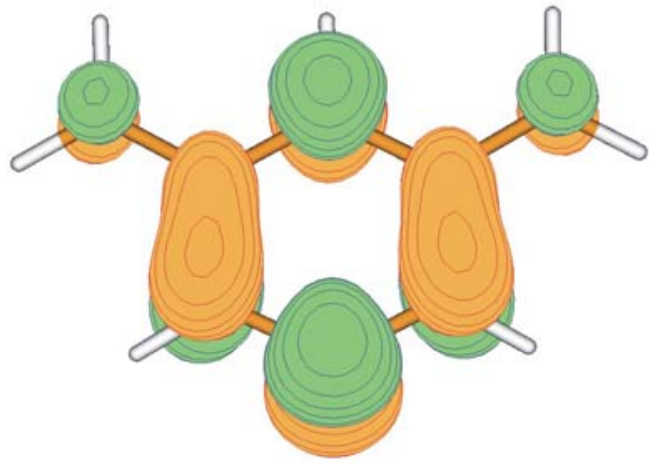

$5 b_{1}$ 
Figure 3S.

The active orbitals of $\mathrm{C}_{6} \mathrm{~N}_{2} \mathrm{H}_{5}$ and $\mathrm{C}_{6} \mathrm{P}_{2} \mathrm{H}_{5}$ at CASSCF/ANO-L.

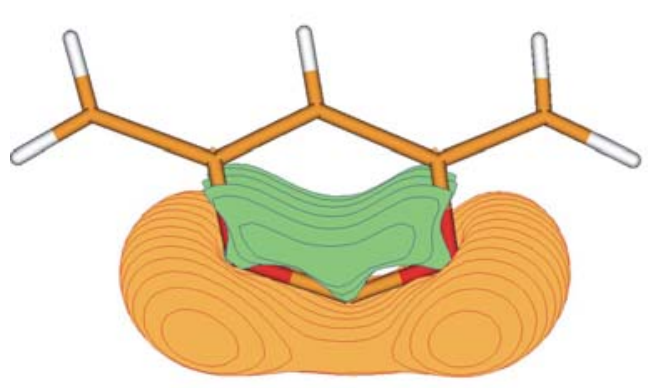

$16 a_{1}$

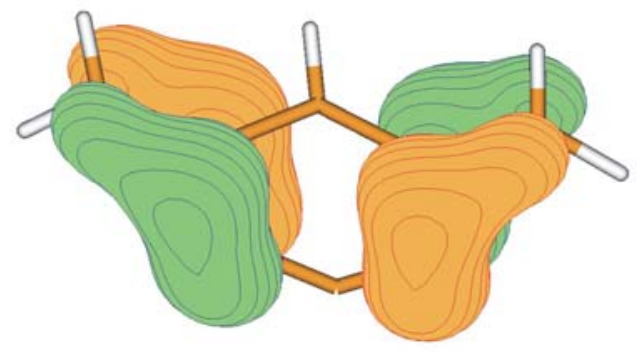

$2 \mathrm{a}_{2}$

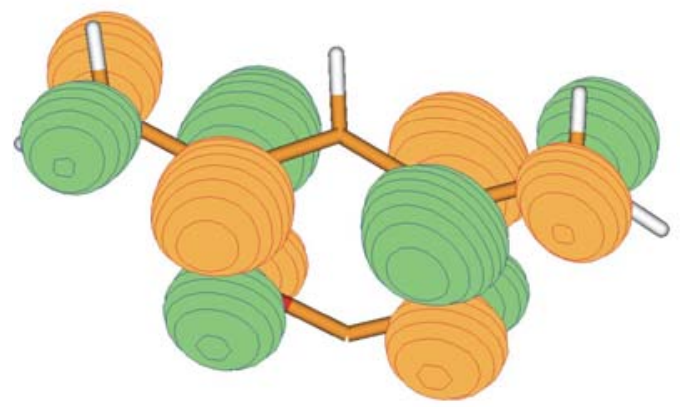

$4 a_{2}$

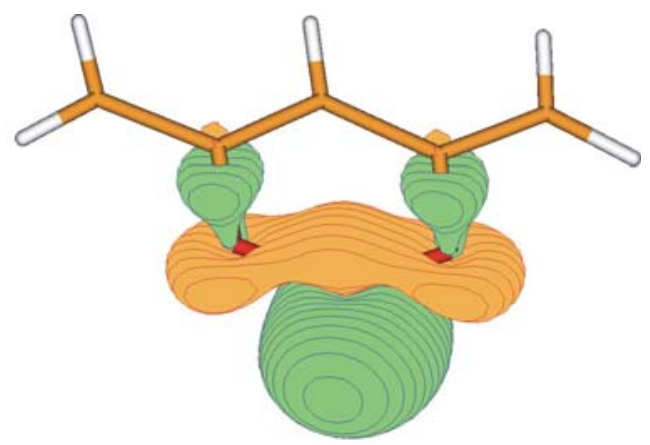

$17 a_{1}$

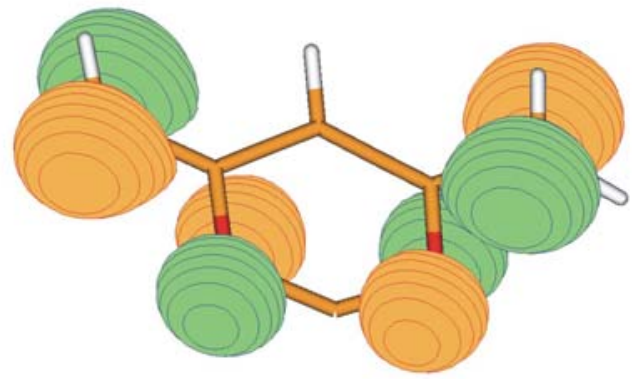

$3 \mathrm{a}_{2}$

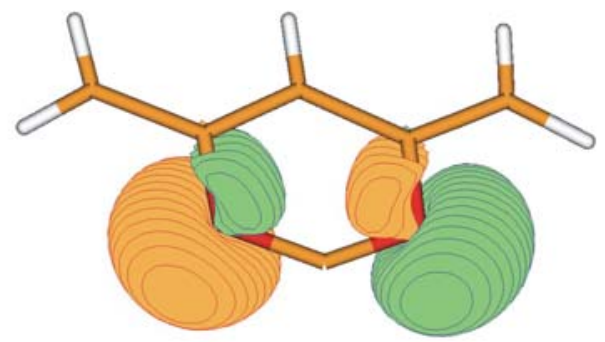

$13 b_{2}$ 
Figure 3S. (Continued)
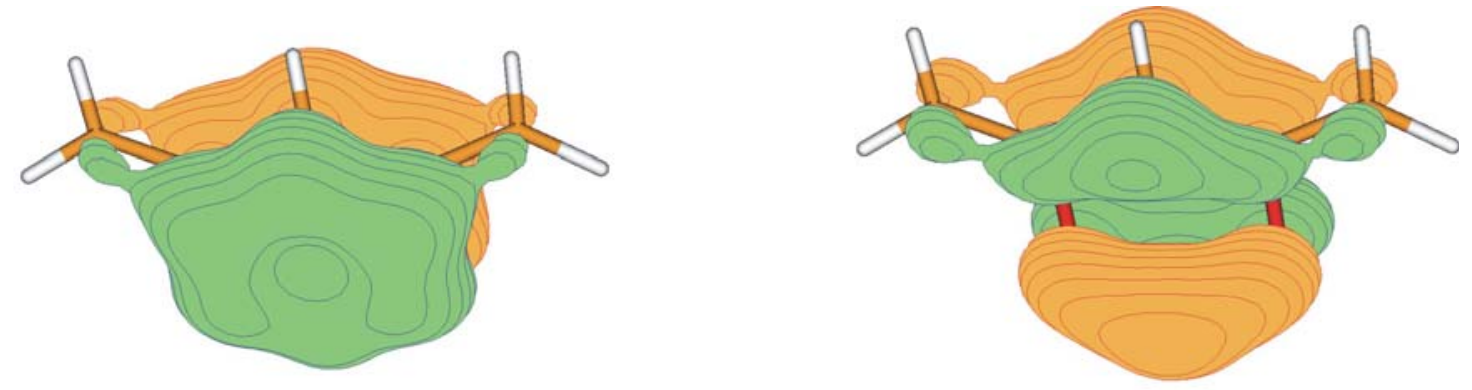

$2 b_{1}$

$3 b_{1}$
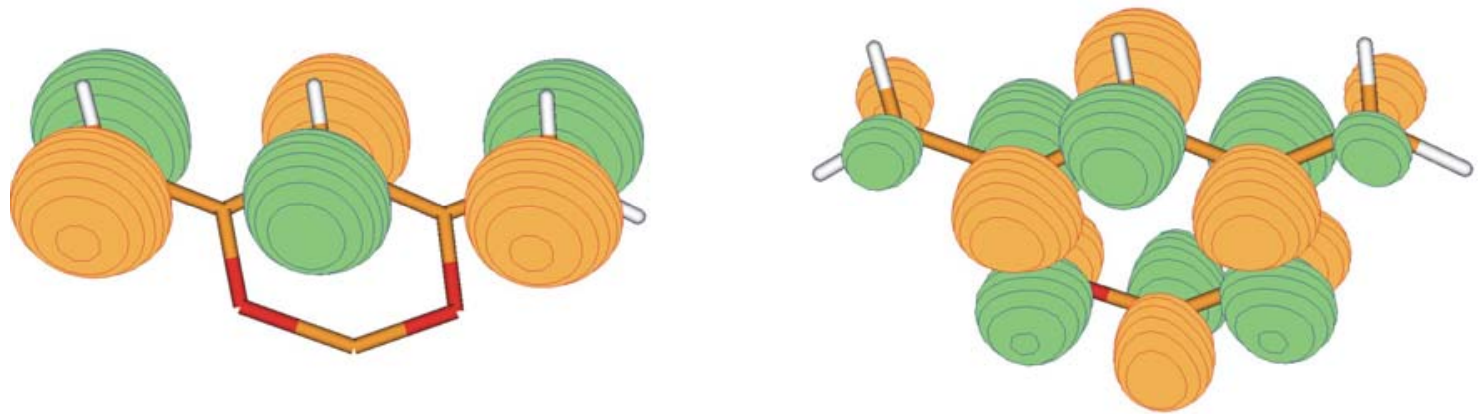

$4 b_{1}$

$5 b_{1}$

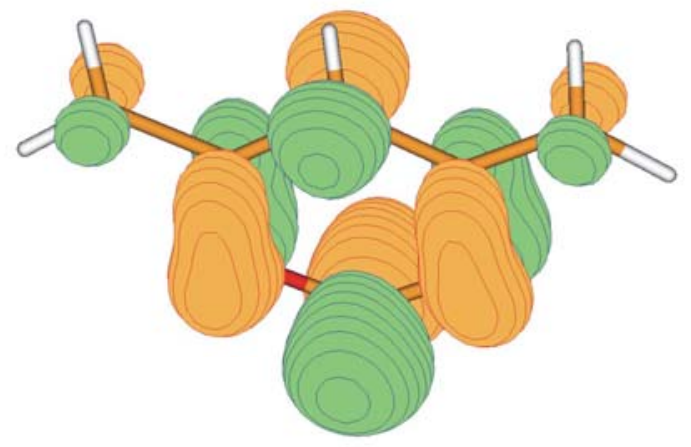

$6 b_{1}$ 
The Cartesian coordinates of the two low lying states of DMX, DMX-N, DMX-P, MX and $\mathrm{MX}^{\circ+}$ at CASSCF/ANO-L.

\begin{tabular}{rrrr} 
& \multicolumn{3}{c}{$\mathbf{D M X}\left({ }^{\mathbf{2}} \mathbf{B}_{2}\right)$} \\
H & 0.000000 & 0.000000 & -2.066098 \\
$\mathrm{H}$ & 0.000000 & -3.395140 & -0.501728 \\
$\mathrm{H}$ & 0.000000 & 3.395140 & -0.501728 \\
$\mathrm{H}$ & 0.000000 & -2.473738 & -2.089036 \\
$\mathrm{H}$ & 0.000000 & 2.473738 & -2.089036 \\
$\mathrm{H}$ & 0.000000 & 2.142990 & 1.675307 \\
$\mathrm{H}$ & 0.000000 & -2.142990 & 1.675307 \\
$\mathrm{C}$ & 0.000000 & 0.000000 & 1.745328 \\
$\mathrm{C}$ & 0.000000 & 1.226299 & 1.120478 \\
$\mathrm{C}$ & 0.000000 & -1.226299 & 1.120478 \\
$\mathrm{C}$ & 0.000000 & 1.247462 & -0.315101 \\
$\mathrm{C}$ & 0.000000 & -1.247462 & -0.315101 \\
$\mathrm{C}$ & 0.000000 & 0.000000 & -0.993186 \\
$\mathrm{C}$ & 0.000000 & -2.456387 & -1.017802 \\
$\mathrm{C}$ & 0.000000 & 2.456387 & -1.017802
\end{tabular}

$\operatorname{DMX}\left({ }^{4} \mathbf{B}_{2}\right)$

$\begin{array}{rrrr}\mathrm{H} & 0.000000 & 0.000000 & -2.063923 \\ \mathrm{H} & 0.000000 & -3.395329 & -0.503098 \\ \mathrm{H} & 0.000000 & 3.395329 & -0.503098 \\ \mathrm{H} & 0.000000 & -2.472788 & -2.089914 \\ \mathrm{H} & 0.000000 & 2.472788 & -2.089914 \\ \mathrm{H} & 0.000000 & 2.142338 & 1.676478 \\ \mathrm{H} & 0.000000 & -2.142338 & 1.676478 \\ \mathrm{C} & 0.000000 & 0.000000 & 1.741788 \\ \mathrm{C} & 0.000000 & 1.226046 & 1.121226 \\ \mathrm{C} & 0.000000 & -1.226046 & 1.121226 \\ \mathrm{C} & 0.000000 & 1.245885 & -0.314274 \\ \mathrm{C} & 0.000000 & -1.245885 & -0.314274 \\ \mathrm{C} & 0.000000 & 0.000000 & -0.990967 \\ \mathrm{C} & 0.000000 & -2.456398 & -1.018718 \\ \mathrm{C} & 0.000000 & 2.456398 & -1.018718\end{array}$




\section{DMX-N ( $\left.{ }^{2} \mathbf{B}_{2}\right)$}

$\begin{array}{rrrr}\mathrm{H} & 0.000000 & 0.000000 & -2.087323 \\ \mathrm{H} & 0.000000 & -3.365651 & -0.338903 \\ \mathrm{H} & 0.000000 & 3.365651 & -0.338903 \\ \mathrm{H} & 0.000000 & -2.558820 & -2.000691 \\ \mathrm{H} & 0.000000 & 2.558820 & -2.000691 \\ \mathrm{C} & 0.000000 & 0.000000 & 1.595497 \\ \mathrm{C} & 0.000000 & 1.218180 & -0.305270 \\ \mathrm{C} & 0.000000 & -1.218180 & -0.305270 \\ \mathrm{C} & 0.000000 & 0.000000 & -1.016517 \\ \mathrm{C} & 0.000000 & -2.476690 & -0.933303 \\ \mathrm{C} & 0.000000 & 2.476690 & -0.933303 \\ \mathrm{~N} & 0.000000 & 1.187110 & 1.056820 \\ \mathrm{~N} & 0.000000 & -1.187110 & 1.056820\end{array}$

DMX-N ( $\left.{ }^{4} B_{2}\right)$

$\begin{array}{rrrr}\mathrm{H} & 0.000000 & 0.000000 & -2.086202 \\ \mathrm{H} & 0.000000 & -3.364974 & -0.338604 \\ \mathrm{H} & 0.000000 & 3.364974 & -0.338604 \\ \mathrm{H} & 0.000000 & -2.558703 & -2.000731 \\ \mathrm{H} & 0.000000 & 2.558703 & -2.000731 \\ \mathrm{C} & 0.000000 & 0.000000 & 1.592482 \\ \mathrm{C} & 0.000000 & 1.216636 & -0.305751 \\ \mathrm{C} & 0.000000 & -1.216636 & -0.305751 \\ \mathrm{C} & 0.000000 & 0.000000 & -1.015383 \\ \mathrm{C} & 0.000000 & -2.476326 & -0.933400 \\ \mathrm{C} & 0.000000 & 2.476326 & -0.933400 \\ \mathrm{~N} & 0.000000 & 1.184144 & 1.058062 \\ \mathrm{~N} & 0.000000 & -1.184144 & 1.058062\end{array}$




\section{DMX-P $\left({ }^{2} \mathbf{B}_{2}\right)$}

$\begin{array}{rrrr}\mathrm{H} & 0.000000 & 0.000000 & -2.444199 \\ \mathrm{H} & 0.000000 & -3.403270 & -1.145001 \\ \mathrm{H} & 0.000000 & 3.403270 & -1.145001 \\ \mathrm{H} & 0.000000 & -2.339773 & -2.636795 \\ \mathrm{H} & 0.000000 & 2.339773 & -2.636795 \\ \mathrm{C} & 0.000000 & 0.000000 & 1.599532 \\ \mathrm{C} & 0.000000 & 1.290232 & -0.763086 \\ \mathrm{C} & 0.000000 & -1.290232 & -0.763086 \\ \mathrm{C} & 0.000000 & 0.000000 & -1.367492 \\ \mathrm{C} & 0.000000 & -2.417373 & -1.566330 \\ \mathrm{C} & 0.000000 & 2.417373 & -1.566330 \\ \mathrm{P} & 0.000000 & 1.600186 & 1.020341 \\ \mathrm{P} & 0.000000 & -1.600186 & 1.020341\end{array}$

\section{DMX-P $\left({ }^{4} \mathbf{B}_{2}\right)$}

$\begin{array}{rrrr}\mathrm{H} & 0.000000 & 0.000000 & -2.442940 \\ \mathrm{H} & 0.000000 & -3.403329 & -1.145576 \\ \mathrm{H} & 0.000000 & 3.403329 & -1.145576 \\ \mathrm{H} & 0.000000 & -2.339443 & -2.637041 \\ \mathrm{H} & 0.000000 & 2.339443 & -2.637041 \\ \mathrm{C} & 0.000000 & 0.000000 & 1.589213 \\ \mathrm{C} & 0.000000 & 1.290448 & -0.763034 \\ \mathrm{C} & 0.000000 & -1.290448 & -0.763034 \\ \mathrm{C} & 0.000000 & 0.000000 & -1.366228 \\ \mathrm{C} & 0.000000 & -2.417312 & -1.566563 \\ \mathrm{C} & 0.000000 & 2.417312 & -1.566563 \\ \mathrm{P} & 0.000000 & 1.596323 & 1.022172 \\ \mathrm{P} & 0.000000 & -1.596323 & 1.022172\end{array}$




\section{$\operatorname{MX}\left({ }^{3} \mathbf{B}_{2}\right)$}

$\begin{array}{lrrr}\mathrm{C} & 0.000000 & 0.000000 & 0.000000 \\ \mathrm{C} & 0.000000 & 0.000000 & 1.415961 \\ \mathrm{C} & 1.266231 & 0.000000 & 2.079507 \\ \mathrm{C} & 2.447013 & 0.000000 & 1.348304 \\ \mathrm{C} & 2.434335 & 0.000000 & -0.040488 \\ \mathrm{C} & 1.196964 & 0.000000 & -0.756412 \\ \mathrm{C} & -1.197994 & 0.000000 & 2.142750 \\ \mathrm{C} & 1.171360 & 0.000000 & -2.157396 \\ \mathrm{H} & -0.939990 & 0.000000 & -0.517918 \\ \mathrm{H} & 2.078801 & 0.000000 & -2.726897 \\ \mathrm{H} & -1.194647 & 0.000000 & 3.214090 \\ \mathrm{H} & 0.243693 & 0.000000 & -2.693482 \\ \mathrm{H} & -2.146750 & 0.000000 & 1.644937 \\ \mathrm{H} & 1.295907 & 0.000000 & 3.151316 \\ \mathrm{H} & 3.356238 & 0.000000 & -0.587983 \\ \mathrm{H} & 3.403014 & 0.000000 & 1.875066\end{array}$

\section{$\operatorname{MX}\left({ }^{1} A_{1}\right)$}

$\begin{array}{lrrr}\text { C } & 0.000000 & 0.000000 & 0.000000 \\ \mathrm{C} & 0.000000 & 0.000000 & 1.400961 \\ \mathrm{C} & 1.241929 & 0.000000 & 2.069559 \\ \mathrm{C} & 2.428952 & 0.000000 & 1.347497 \\ \mathrm{C} & 2.413263 & 0.000000 & -0.041801 \\ \mathrm{C} & 1.188638 & 0.000000 & -0.741590 \\ \mathrm{C} & -1.244743 & 0.000000 & 2.145187 \\ \mathrm{C} & 1.161201 & 0.000000 & -2.191592 \\ \mathrm{H} & -0.938998 & 0.000000 & -0.520924 \\ \mathrm{H} & 2.070530 & 0.000000 & -2.756191 \\ \mathrm{H} & -1.242445 & 0.000000 & 3.215535 \\ \mathrm{H} & 0.232263 & 0.000000 & -2.723514 \\ \mathrm{H} & -2.187738 & 0.000000 & 1.638603 \\ \mathrm{H} & 1.264921 & 0.000000 & 3.142161 \\ \mathrm{H} & 3.335457 & 0.000000 & -0.590035 \\ \mathrm{H} & 3.367095 & 0.000000 & 1.867947\end{array}$




\section{$\mathbf{M X}^{\circ+}\left({ }^{2} \mathbf{B}_{1}\right)$}

$\begin{array}{rrrr}\mathrm{H} & 0.000000 & 0.000000 & -2.146230 \\ \mathrm{H} & 0.000000 & -3.372262 & -0.424650 \\ \mathrm{H} & 0.000000 & 3.372262 & -0.424650 \\ \mathrm{H} & 0.000000 & -2.551528 & -2.064242 \\ \mathrm{H} & 0.000000 & 2.551528 & -2.064242 \\ \mathrm{H} & 0.000000 & 2.121526 & 1.601261 \\ \mathrm{H} & 0.000000 & -2.121526 & 1.601261 \\ \mathrm{H} & 0.000000 & 0.000000 & 2.840744 \\ \mathrm{C} & 0.000000 & 0.000000 & 1.770277 \\ \mathrm{C} & 0.000000 & 1.190882 & 1.068156 \\ \mathrm{C} & 0.000000 & -1.190882 & 1.068156 \\ \mathrm{C} & 0.000000 & 1.227983 & -0.374111 \\ \mathrm{C} & 0.000000 & -1.227983 & -0.374111 \\ \mathrm{C} & 0.000000 & 0.000000 & -1.075328 \\ \mathrm{C} & 0.000000 & -2.466673 & -0.996136 \\ \mathrm{C} & 0.000000 & 2.466673 & -0.996136 \\ & & \end{array}$

$\begin{array}{rrrr}\mathrm{H} & 0.000000 & 0.000000 & -2.096098 \\ \mathrm{H} & 0.000000 & -3.364559 & -0.631173 \\ \mathrm{H} & 0.000000 & 3.364559 & -0.631173 \\ \mathrm{H} & 0.000000 & -2.353780 & -2.160428 \\ \mathrm{H} & 0.000000 & 2.353780 & -2.160428 \\ \mathrm{H} & 0.000000 & 2.138765 & 1.644600 \\ \mathrm{H} & 0.000000 & -2.138765 & 1.644600 \\ \mathrm{H} & 0.000000 & 0.000000 & 2.855096 \\ \mathrm{C} & 0.000000 & 0.000000 & 1.784019 \\ \mathrm{C} & 0.000000 & 1.212928 & 1.107317 \\ \mathrm{C} & 0.000000 & -1.212928 & 1.107317 \\ \mathrm{C} & 0.000000 & 1.241288 & -0.332767 \\ \mathrm{C} & 0.000000 & -1.241288 & -0.332767 \\ \mathrm{C} & 0.000000 & 0.000000 & -1.024411 \\ \mathrm{C} & 0.000000 & -2.395440 & -1.089895 \\ \mathrm{C} & 0.000000 & 2.395440 & -1.089895\end{array}$

